Recherches en didactique des langues et des cultures

Les cahiers de l'Acedle

12-1 | 2015

Prendre la diversité au sérieux en didactique / didactologie des langues

\title{
La Chinafrique en contextes universitaires
}

Une francophonie non prévue?

David Bel et Valentin Feussi

\section{OpenEdition}

\section{Journals}

Édition électronique

URL : http://journals.openedition.org/rdlc/366

DOI : $10.4000 /$ rdlc.366

ISSN : $1958-5772$

Éditeur

ACEDLE

Référence électronique

David Bel et Valentin Feussi, "La Chinafrique en contextes universitaires », Recherches en didactique des langues et des cultures [En ligne], 12-1 | 2015, mis en ligne le 01 juin 2015, consulté le 23 juin 2020 URL : http://journals.openedition.org/rdlc/366 ; DOI : https://doi.org/10.4000/rdlc.366

Ce document a été généré automatiquement le 23 juin 2020.

\section{(c) ()}

Recherches en didactique des langues et des cultures is licensed under a Creative Commons AttributionNonCommercial-NoDerivatives 4.0 International License 


\section{La Chinafrique en contextes universitaires}

Une francophonie non prévue?

David Bel et Valentin Feussi

\section{Introduction}

1 Depuis plusieurs années, les relations entre l'Afrique et la Chine connaissent un développement considérable, au point que l'expression Chinafrique semble être entrée dans le vocabulaire courant, expression pourtant bien ambivalente, surtout si l'on se réfère au modèle sur lequel elle a été calquée (Françafrique) ${ }^{1}$. Si c'est l'aspect économique qui est le plus souvent mis en avant, cette rencontre entre tout un continent et le géant démographique asiatique ne se limite pas à cette dimension. Les liens académiques se multiplient également, puisque de nombreux étudiants africains viennent faire des études en Chine et que les Instituts Confucius sont de plus en plus nombreux à s'implanter en Afrique. Or, cette rencontre est encore peu interrogée, notamment du point de vue des enjeux de la présence de langues (le chinois en l'occurrence), voire des vécus de la diversité impliquée, ici représentée et de façon inattendue par la francophonie. Quels rapports entre Chinafrique et francophonie ? La mise en lien de ces espaces, que ce soit sur le plan géopolitique, linguistique ou culturel conduit à des mises en tension de points de vue variés, comme nous allons le voir dans la suite de cet article. Nous nous proposons de commencer par situer le cadre expérientiel d'émergence de cette réflexion. Par la suite, nous présenterons la construction notionnelle de la francophonie en Chine avant de réfléchir à sa perception en Chine ainsi qu'à la place de l'Afrique dans cette construction. Nous terminerons alors par une interrogation sur les enjeux politiques et stratégiques de la Chinafrique. 


\section{Positionnements académiques et institutionnels, parcours et projets professionnels des individus: quelle francophonie?}

2 L'idée de ce projet est née de rencontres académiques organisées à l'Université Normale de Chine du Sud (désormais UNCS) à Canton, dans le cadre du développement de son jeune département de français². D'emblée orienté vers le monde francophone (et donc pas seulement vers la France), ce département s'est retrouvé face à deux questions accompagnant notamment l'enseignement du français: qu'est-ce que la francophonie et comment l'enseigner? Il est en effet devenu évident, au moment où les enseignants élaboraient les cours les uns après les autres, mais aussi au moment où se mettaient en place les programmes de coopération à l'international, qu'une telle réflexion ne pouvait être évitée. C'est ce qui nous (auteurs et collaborateurs de cet article) a amenés à y réfléchir, dans le cadre du projet DIFFODIA ${ }^{3}$. Cette réflexion, d'abord mise en œuvre à partir d'un cours de "francophonie " proposé à l'UNCS, s'appuie également sur des croisements d'expériences impliquant différentes formes d'échanges avec des étudiants, des enseignants et des responsables académiques, l'objectif général étant de comprendre certains phénomènes liés à la francophonie, apparus de façon inattendue.

\section{Une francophonie inattendue}

Pour comprendre le caractère inattendu de cette francophonie, il convient de prendre en considération des éléments d'expériences et de parcours individuels de personnes impliquées à des degrés divers dans cette réflexion. Citons d'une part celle de la directrice de l'International Business College (à l'origine de la création de la spécialité de français $\left.{ }^{4}\right)$ qui, sur le plan de la gestion administrative universitaire, a une image très positive de l'international. D'autre part, celle du directeur du département de français (coauteur de cet article) qui, au moment de sa nomination, n'avait pas saisi que son recrutement constituait un signe fort d'ouverture de la part de l'université. Ayant fait le choix de quitter la France, en partie par rejet, et d'émigrer au Québec - avant de, finalement se retrouver fortuitement en Chine -, il avait clairement développé une volonté de ne pas recréer une 'petite France' à travers ce département de français naissant, ce qui a eu comme conséquence une orientation des enseignements vers le monde francophone. L'attraction vers une diversité aux contours pourtant bien flous joua-t-elle en partie un rôle de repoussoir par rapport à la France ? Toujours est-il que le recrutement des enseignants du département a, au début, suivi une logique : ne pas laisser aux Français le « monopole » des postes d'enseignants « étrangers ».

4 De même, la recherche de partenaires pour ce département naissant s'est tournée vers le Québec, la Belgique, la Suisse et l'Afrique. Enfin, cela a conduit à l'ouverture, en $3^{e}$ année, de deux cours sur un semestre chacun sur la francophonie puis sur les Afriques francophones ${ }^{5}$.

5 L'entretien réalisé avec la directrice (supra) révèle chez elle une francophilie centrée sur la France $^{6}$ et une volonté d'ancrage à l'international sans lien apparent à la francophonie. C'est par pragmatisme (gain symbolique d'une ouverture vers d'autres parties du monde, inconnues, peut-être différentes, où on parle le français) que l'UNCS 
va ainsi adhérer à l'Agence Universitaire de la Francophonie et développer une coopération en dehors de la France, notamment au Canada et au Liban. Cette démarche conduit ainsi à une "francophonie surprise ", du moins pour l'UNCS, laquelle s'élargit dans le cadre du cours sur la francophonie. Ce cours propose en effet une déclinaison africaine de la francophonie, vécue par les étudiants de l'UNCS à travers des rencontres avec des francophones non-Français à Canton (commerçants et migrants originaires d'Afrique ${ }^{7}$ ). Ainsi, la présence de cette « francophonie " à l'UNCS apparaît comme une conséquence inattendue d'une politique d'« ouverture » à l'international.

Cette ouverture conduit à une transformation de regards sur la gestion de ressources humaines à l'université et à la prise en compte de l'impact de différences dans les stéréotypes liés aux nationalités. La désignation du directeur du département de français (supra), qui se fait sur une ambigüité mettant en lien images et stéréotypes, en est une illustration : faire preuve d'ouverture et de modernité en nommant un Français à la tête d'un département universitaire ${ }^{8}$ ("profiter» en même temps de l'image positive de la langue française et de la France, quasi systématiquement liées et positivement perçues en Chine), tout en s'assurant de l' « efficacité » du choix".

7 C'est donc presque sur un "malentendu", au final profitable pour tous, que ce département de français s'est tourné vers la francophonie, tout en révélant des aspects de la diversité liés aux expériences individuelles considérées comme modalité de gestion d'institutions universitaires.

\section{Des parcours fondés sur des représentations}

8 La place des représentations permet de mieux comprendre cette rencontre inattendue entre le français, la diversité francophone et l'Afrique.

En interprétant les éléments de corpus constitués des propos recueillis auprès des étudiants à l'UNCS, nous observons que les différentes représentations de la francophonie se font en lien avec des espaces précis : la France ou le Québec, considérés comme des cadres de visibilisation du français, langue pour laquelle certains ont développé une sensibilité particulière. C'est ainsi que pour une des étudiantes chinoises rencontrées, le français «est (...) élégant, le rythme est très rapide, et l'intonation est intéressante ».

10 La francophonie prend ici les couleurs de ce que Senghor ${ }^{10}$ appelait " francité », c'est-àdire « l'esprit français, [...] une logique élégante » et de "clarté » : elle est alors réduite à la langue et à la civilisation françaises. On a l'impression dans ce contexte que la francité renvoie à une vision stéréotypée qui oublie de rappeler les enjeux qui accompagnent les projets liés à l'apprentissage du français. Les étudiants de l'UNCS que nous avons rencontrés se projettent en tout cas en francophonie grâce à une image très magnifiée du français, perçu du point de vue de sa pureté. D'où l'étonnement face à l'émergence de l'Afrique, continent qu'ils connaissent surtout à travers des représentations très péjoratives (pauvreté, confits de natures diverses, retard économique et infrastructurel, espace qui a permanemment besoin d'«aide humanitaire...» (Li, 2007: 127)), plus que comme lieu de présence de la langue française.

11 Si l'on se tourne maintenant vers des étudiants camerounais ${ }^{11}$ rencontrés pour cette recherche et qui préparent des séjours en Chine, on observe que la francophonie ne fait pas non plus partie des attentes de formation par rapport à la Chine. Leurs projets 
académiques et/ou professionnels s'articulent autour du chinois et sont également accompagnés de représentations de la Chine sans aucun rapport avec le français et / ou la francophonie. Ces images du chinois et de la Chine sont plutôt valorisantes et portées en grande partie par des produits culturels que sont notamment le cinéma et la bande dessinée :

Depuis l'enfance, je suis fasciné par les bandes dessinées et la culture orientale, mais je vais surtout m'inscrire à l'Institut Confucius, à la rentrée prochaine, pour ensuite suivre des études de médecine en Chine. (Étudiant camerounais 1).

La dimension économique apparaît également comme féconde dans les discours des locuteurs, qui représentent la Chine comme un pays émergent (donc attirant), dont les performances économiques sont désormais remarquables: «La Chine, pays ouvert, pays des relations, des transactions, donc c'est cette image que j'ai de la Chine». ( Étudiant camerounais 2).

Voilà comment on pourrait en partie interpréter l'apprentissage du chinois: l'acquisition de compétences non linguistiques, contributives d'un développement professionnel en construction. En fait, l'apprentissage de langues accompagne toujours un/des projet(s) qui, dans le fond, n'est/ne sont pas linguistique(s). Nous ne voulons pas trop développer cet aspect plus sociolinguistique ${ }^{12}$, notre objectif étant surtout de rappeler que ces projets académiques et personnels élaborés à partir des langues, constituent des moyens efficients pour problématiser d'autres éléments d'expériences, pour appréhender cette ouverture à la fois institutionnelle et expérientielle qui conduit vers des considérations plus francophones. Mais peut-on, pour autant, considérer que la Chine est concernée par cette « solidarité » (Deniau, 2003: 10) dont se revendique la francophonie?

\section{La francophonie en Chine : une construction notionnelle récente et encore en gestation}

\section{La francophonie : une notion peu traduite et peu utilisée}

Ces discours d'étudiants sur la francophonie ne sont, en fait, pas si étonnants, vu que la francophonie est une notion apparue récemment en Chine (et dont la traduction en chinois pose problème - voir infra). Pour mieux nous en rendre compte, nous nous sommes intéressés au volume d'utilisation, dans des articles de recherche, des termes francophonie et francophone. Pour cela, nous avons choisi la revue Synergies Chine ${ }^{13}$, seule revue d'expression française en Chine portant sur les langues et leur enseignement. Les articles sont majoritairement, mais pas exclusivement, écrits par des enseignants et chercheurs chinois.

Il en ressort que francophone et francophonie sont peu utilisés et renvoient respectivement à « qui parle français » et « où l'on parle français ${ }^{14}$ » (un endroit: pays, province, région... ou un contexte), sans aucune référence explicitée à une quelconque diversité. En outre, le sens qui est conféré à francophonie est ambivalent. Il peut désigner les pays qui ont "la langue française en partage " comme aime à le définir l'Organisation Internationale de la Francophonie (désormais OIF) en se gardant bien de préciser ce qui est partagé exactement, comment et jusqu'à quel point. Il peut également désigner des espaces où l'on parle le français, sens présent depuis les 
travaux du géographe O. Reclus (1886). Cette ambivalence du terme francophonie dans les discours de scientifiques en vient, en pratique, lorsqu'il est utilisé, à désigner tous ces pays, en dehors de la France, afin justement de dire qu'on ne parle pas spécifiquement de la France (ce à quoi l'adjectif français fait clairement référence). A tel point que francophonie est parfois devenu synonyme de ce que Valdman (1979) appelait la «francophonie hors de France $»^{15}$ (comme si la France était en dehors de la francophonie). Toutefois, la situation semble évoluer depuis quelques temps.

\section{Une prise en compte récente?}

La traduction du terme francophonie en chinois apparaît comme un obstacle majeur à la prise en compte de la diversité francophone. Comment faire en sorte qu'il se distingue de l'adjectif français, et donc de la référence exclusive à la France ? On peut le traduire à la fois :

1. sous une forme paraphrastique à la fonction explicative : 法语世界 ; 法语国家和地区及其 文化的统称 (《le monde francophone », « l'ensemble des pays et régions francophones avec leurs cultures » au sens large du terme).

Ou bien

1. sous la forme de syntagmes qui peuvent désigner:

- «études francophones»: 以法语作为工作语言的科学研究 (études dont le français est la langue de travail); 与法语国家和地区相关的科学研究 (études par rapport aux pays et régions de la Francophonie - c'est-à-dire membres de l'OIF)

• « espaces francophones 》 (法国国家和地区 ; 法语区 ; 法语空间).

Dans les écrits en français, nous remarquons une évolution de la situation. Dans les articles de Synergies Chine, le terme francophone est davantage utilisé dans les deux derniers numéros (alors que le thème ne se prête pas particulièrement à des articles sur la francophonie $\left.{ }^{16}\right)$. En y regardant de plus près, cette évolution est surtout due au fait que la liste des contributeurs s'internationalise de plus en plus (voir le n. 8 en particulier). Il s'agit néanmoins de la traduction d'une prise en compte - tardive, timide mais réelle - d'une diversité francophone. Cette tendance est-elle liée à une gêne croissante face à l'utilisation exclusive de l'adjectif «français», qui fait trop systématiquement penser à la seule France?

Plus significatif est le développement récent de centres de recherche sur la francophonie. Encore peu nombreux et peut-être pas très actifs (pour certains au moins), leur développement témoigne d'une volonté, au moins officielle, de mieux comprendre la diversité du monde francophone et notamment de l'Afrique qui, il faut le remarquer, n'apparaît que très rarement dans les articles de Synergie Chine. Leur création apparaît ainsi comme une réponse à des questionnements multipliés avec l'implication croissante de la Chine dans une dynamique de la mondialisation, notamment en Afrique. Ainsi, si les instituts de recherche sur l'Afrique sont relativement anciens et nombreux, à l'instar de l'Institut des études ouest-asiatiques et africaines de l'Académie des sciences sociales de Chine fondé en 1961, plusieurs lieux de recherche portant spécifiquement sur la francophonie et travaillant essentiellement en chinois et en français sont apparus beaucoup plus récemment, comme le Centre de recherche sur la Francophonie de l'Institut de diplomatie ${ }^{17}$ (2009) et le Centre de recherches sur des pays francophones de l'Université Fudan ${ }^{18}$ (2010). 


\section{Quelles perceptions et quelle place pour la francophonie et l'Afrique dans le monde universitaire chinois?}

\section{La diversité: un souhait}

Dans la logique d'un refrain caractéristique du positionnement de l'OIF, Wolton (2012, 327) affirme que la francophonie ouvre un espace de dialogue « en faveur de la diversité linguistique et culturelle et du dialogue entre les cultures ». Cette représentation (qui considère l'espace francophone par essence comme porteur de diversité et de pluralité), bien que discutable, est cependant largement répandue. Les articles de Synergies Chine ne considèrent malheureusement pas cette dimension plurielle, même sous cette forme stéréotypée : c'est toujours d'un dialogue sino-français dont il est question, car « depuis longtemps, aux yeux de bien des Chinois, et même de beaucoup d'Asiatiques, l'apprentissage du français est associé uniquement à l'apprentissage de la culture française. » (Li, 2007: 125).

Parce que le phénomène "francophonie » est méconnu des enseignants, c'est en toute logique que l'on retrouve cette ignorance du fait francophone parmi leurs étudiants : Les étudiants chinois ignorent la culture des "Français d'Amérique ", les Québécois, celles des Belges et des Suisses et du vaste territoire francophone africain, et même leurs professeurs ne la connaissent que très peu. (Li, 2007 : 125).

Et puis, après avoir suivi tous nos cours, si les élèves peuvent acquérir suffisamment de connaissances sur la langue française, et notamment sur le pays le plus concerné par cette langue: la France, ils ne connaissent que très peu de choses sur les autres pays et régions francophones. Pourtant, « il n'y pas que les Français qui parlent français». Au fur et à mesure du développement des relations entre la Chine et l'Afrique, le Québec, la Belgique, la Suisse, etc., l'importance des connaissances sur la culture de ces pays et régions se fait sentir. (Chen, $2008: 64)$.

Dans le même sens, Pernet-Liu (2013) s'intéresse aux mémoires de fin d'études d'étudiants chinois de français de deux universités de Pékin ${ }^{19}$ pour constater la rareté de sujets portant sur la francophonie et la prédominance d'une approche comparative Chine-France.

Les termes francophone et francophonie ne sont donc pas abordés dans une perspective diversitaire. Plus abondement utilisés par Li dans deux articles portant sur le cours «francophonie », un des objectifs de leur présence est certes de fournir des conseils pédagogiques, de partager des expériences, mais surtout de lancer un appel pour encourager ses collègues chinois à connaître et à faire connaître la francophonie :

En tant que francophones, asiatiques ou américains, européens ou africains, il nous est impossible de négliger le sens du mot «francophonie», ainsi que LES cultures qui s'y attachent. (Li, $2007:$ 125).

\section{Et l'Afrique dans tout ça?}

27 Le fait d'associer, presque de manière exclusive, langue et culture françaises n'est pas fortuit dans les situations universitaires chinoises. Il semble bien que, pour un but d'affichage, il y ait un intérêt pour les professeurs de français (et pour les universités) à 
profiter de l'image très positive de la France et de la culture française en Chine, que l'on retrouve dans nombre d'enquêtes (voir par exemple Hu ou Xie), et a contrario, peu d'intérêt à associer le français et l'Afrique, du fait de l'image presque systématiquement négative de cette dernière.

Bien que Li ait une vision globalement réductrice de l'Afrique, on ne peut nier que son propos détonne, dans un paysage où les appels à la diversité sont rares ${ }^{20}$, et qu'il représente une avancée par rapport à nombre de ses collègues, surtout au moment où il a été publié (2007). Effectivement, on constate que dans notre corpus d'articles de Synergies Chine, le terme Afrique apparait, en huit ans, huit fois et le terme africain deux fois, et qu'ils sont surtout utilisés par Li $(2007,2010)$.

En Chine, le Programme national ${ }^{21}$ encadre les études de spécialité de français pour les quatre années que dure le premier cycle universitaire en Chine. On y remarque un cours facultatif de présentation de la francophonie, en troisième année. Lorsqu'il est ouvert, il est orienté vers ce que l'OIF appelle "Francophonie du Nord", à savoir la France, la Suisse, la Belgique et le Québec. On peut constater que l'Afrique est ignorée. Si on se fie à l'expérience d'un collègue de Canton, les seuls moments où cet espace est évoquée dans les cours sont les «quelques minutes de présentation de la carte des pays francophones" en début de semestre ou éventuellement durant la partie du cours portant sur l'OIF. Ainsi, il est très probable que des étudiants chinois, apprenant le français comme spécialité universitaire ou comme option, n'aient quasiment jamais entendu parler de l'Afrique francophone au cours de leurs études.

Pourtant, les pays africains francophones sont de plus en plus présents dans les projets avec différents acteurs chinois (de même que l'Afrique est de plus en plus présente dans les discours de la francophonie institutionnelle), ce qui légitime davantage l'option choisie d'ouvrir une spécialité de français dans les universités chinoises, mais également la mise en œuvre au niveau universitaire en Afrique, de projets d'enseignement du chinois, choix qui permet de tisser des liens de coopération entre ces pays et la Chine.

\section{La Chinafrique dans la coopération universitaire}

31 La mise en œuvre des liens entre la Chine et les pays africains peut s'observer sur deux plans: la communication et la coopération internationale. Concernant la communication institutionnelle, nous nous sommes intéressés aux sites Internet officiels des universités. Éléments d'affichage, ils donnent des indications sur leurs positionnements et sur les images qu'elles souhaitent se donner. Aucune des universités chinoises offrant une spécialité de français que nous avons étudiées ${ }^{22}$ ne communique sur les débouchés professionnels possibles en Afrique francophone et très peu évoquent le monde francophone, si ce n'est pour parler de la France, venant ainsi confirmer les impressions précédentes. Pour ce qui concerne la coopération interuniversitaire, les universités des pays africains ne font quasiment pas partie du paysage universitaire chinois.

Toutefois, deux phénomènes observés en Chine peuvent susciter des questionnements. Le premier est la présence de plus en plus importante d'étudiants africains qui suivent souvent des cursus universitaires complets (dans des spécialités comme la médecine, l'informatique et la traduction pour les plus citées dans nos enquêtes), après y avoir appris le chinois. Ces étudiants sont quasiment toujours bénéficiaires d'une bourse du 
gouvernement chinois. Même s'il ne s'agit pas à proprement parler de coopérations entre institutions, la présence de ces étudiants africains souvent francophones sur les campus chinois entraîne une ouverture de facto vers ce continent (en même temps qu'elle en témoigne). Le deuxième phénomène relève de la formation linguistique de ces étudiants et commerçants d'origine africaine dont nous parlions supra. Leurs projets de séjour en Chine les orientent vers des institutions où ils pourraient développer des compétences linguistiques en chinois comme les Instituts Confucius (désormais IC).

Mis en place par le gouvernement chinois pour répondre à ce type de demandes, les IC sont actuellement près de 450 dans le monde, répartis sur cinq continents. Mais l'Afrique ne représente que moins de $10 \%$ de ce total (38, contre 97 aux États-Unis, et près de 150 en Europe ${ }^{23}$ ). La structure est quasiment la même partout : rattachés à une université chinoise et à une université du pays d'accueil, les IC sont essentiellement financés par le gouvernement chinois. L'IC de Yaoundé au Cameroun, par exemple, a été confié par le Hanban ${ }^{24}$ à l'université du Zhejang (une même université peut se voir confier plusieurs instituts) et a été rattaché du côté camerounais à l'Université de Yaoundé II. Il faut noter que le choix de l'université chinoise ne se fait pas nécessairement en fonction de l'existence ou non en son sein d'un département de langues étrangères. Néanmoins, la coopération est essentielle dans ces instituts, puisqu'y sont vécues des expériences de codirection : un directeur chinois envoyé par l'université chinoise de tutelle et un directeur local nommé par l'université d'accueil.

Le contraste est ainsi saisissant entre la méconnaissance de l'Afrique et une réalité économique qui amène de plus en plus d'étudiants chinois à y travailler. On se retrouve dans la situation d'une rencontre non prévue, voire même non désirée (notamment si on considère le monde universitaire), en tout cas, une rencontre fortuite avec l'Afrique. Voilà pourquoi on peut comprendre le cadre de la Chinafrique comme une rencontre avant tout économique. On peut ainsi émettre l'hypothèse que les parcours individuels sont articulés à des perspectives plus globales. C'est pourquoi nous souhaitons terminer cette réflexion en nous tournant vers les enjeux pluriels de la Chinafrique en lien avec la francophonie.

\section{Chinafrique, francophonie: quels enjeux ?}

Pour comprendre cette partie terminale de notre réflexion, il convient de partir de nos expériences et parcours de vie qui articulent Chine, Afrique et francophonie, de revenir vers des éléments des biographies respectives des co-auteurs de cet article. L'un, Camerounais d'origine, enseigne dans une université française avec une expérience de vie au Cameroun puis en France; l'autre, Français, est directeur du département de français de l'UNCS. A ces expériences, on peut associer celle de la responsable de la création du département de français à l'UNCS, chinoise avec une expérience de l'Amérique du Nord. Ce tissage d'expériences (à forte composante mobilitaire) correspond à des mises en tension d'altérités dont l'affichage dans cette réflexion peut être considéré comme bouées à saisir par le lecteur pour se construire une idée de la situation, en s'inspirant de son parcours de vie, ce qui constitue en partie un des objectifs revendiqués du projet DIFFODIA. Elles peuvent se décliner en deux entrées non contradictoires mais qui ne révèlent pas les mêmes enjeux : la politique d'influence et l'implication d'acteurs divers. 


\section{La présence de la Chine en Afrique: une politique d'influence?}

Une de nos interprétations découle de la mise en relief d'un axe politique pour comprendre les projets universitaires en Chine en lien avec la francophonie et surtout l'Afrique, à partir des enjeux pour la Chine. Il en découle alors des éléments de politique d'influence, qui serait un enjeu important pour comprendre la Chinafrique. Focalisons-nous sur l'exemple de la présence du chinois dans certaines institutions universitaires au Cameroun ${ }^{25}$ (et dans le monde). Pour ce faire, partons d'un extrait du texte de présentation des Instituts Confucius, mis en exergue sur le site Internet du Hanban :

As China's economy and exchanges with the world have seen rapid growth, there has also been a sharp increase in the world's demands for Chinese learning. Benefiting from the UK, France, Germany and Spain's experience in promoting their national languages, China began its own exploration through establishing non-profit public institutions which aim to promote Chinese language and culture in foreign countries in 2004 : these were given the name the Confucius Institute.

À la lumière de cet extrait, on comprend que l'exportation du chinois répond à des besoins géostratégiques de la Chine qui s'inspire du modèle de pays européens ayant développé des expériences réussies dans l'expansion linguistique, culturelle et surtout économique. Si nous prenons l'exemple de l'Alliance française, on pourrait alors paraphraser Chaubet (2004) qui la considère comme une diplomatie de la langue, pour dire que la Chine reproduit le même schéma en adoptant des processus qu'elle n'affiche pas, procédé d'évitement qui permet de surfer sur les arguments comme «aide à ». Il s'ensuit une dimension de «non-profit » qui laisse penser que la diffusion du chinois correspond à une demande locale dans chacune des situations envisagées. En fait, les projets académiques institutionnels relèvent plutôt d'une politique d'influence visant à asseoir la domination symbolique de la Chine en Afrique.

Dans les manuels d'apprentissage du chinois, qui tiennent lieu de programme pour l'instant à l'IC de Yaoundé (aucun dispositif curriculaire n'est élaboré pour l'enseignement du chinois en Afrique), le chinois est présenté avec une focalisation sur le mandarin. Sans vouloir discuter de la diversité linguistique de la Chine, le simple fait de considérer le mandarin comme une "putong hua (littéralement langue commune, avec un objectif : promotion d'une image simplificatrice et harmonieuse de la Chine) rappelle la logique classique des États-Nations. Cette situation pourra-t-elle changer? Pour s'en tenir aux situations camerounaises (et africaines), une enseignante de chinois à l'IC de Yaoundé nous indique que certains projets en cours auraient pour objectifs d'élaborer des ouvrages appropriés pour prendre en compte la diversité linguistique en contexte camerounais, tâche qui partirait d'une réflexion sur des programmes à mettre en œuvre pour l'enseignement du chinois au Cameroun et en Afrique. Quelle(s) forme(s) de diversité seront privilégiées dans ce cadre ? Si la langue enseignée dans ces ouvrages reste le mandarin, il y aura des chances que ce projet débouche sur un gommage de la diversité linguistique ${ }^{26}$, comme c'est le cas actuellement. Cette offre de formation s'accompagne en effet d'actions diverses: des bourses d'études proposées pour certaines filières d'études, le "Chinese bridge competition" (un concours international de maîtrise de la langue chinoise) qui constitue une modalité de sélection pour des séjours en Chine, des échanges commerciaux, des projets de travaux publics et 
de formation de cadres du service public, notamment. Pourrait-on penser que ces éléments constituent des ressources au service d'une stratégie d'expansion qui caractériserait d'ailleurs la «nouvelle politique » de Pékin "destinée à servir à la fois ses propres besoins économiques croissants et sa montée en puissance sur la scène mondiale » (Cabestan, 2013: 150) ?

\section{Stratégies des acteurs impliqués} acteur, si puissant soit-il, peut conduire à gommer les stratégies, les parcours, les expériences et les usages des acteurs impliqués, si divers soient-ils. Ces acteurs agissent dans une situation complexe, avec comme objectif de suivre leur propre agenda et comme limite celle de s'adapter aux éléments de structure, parfois très contraignants, comme la politique du gouvernement (mais il y en a bien d'autres). hiérarchisé. En compétition entre elles, elles ne jouent pas toutes dans la même cour, loin s'en faut. Les contraintes qui pèsent sur elles, notamment gouvernementales, ne sont pas de même force. Une université prestigieuse, dirigée directement par Pékin (Ministère de l'éducation) sera beaucoup plus exposée et obligée de suivre les instructions officielles qu'une université de second rang. Néanmoins, elles suivent leur propre agenda et trouvent les moyens de refuser bien des injonctions gouvernementales (voir les résistances de certaines lors des fusions forcées d'universités dans les années 1990-2000 dans Hayhoe (2011) et Bel (2014)). Ceci sans parler d'une privatisation non avouée qu'on pourrait qualifier d'outrancière, de l'enseignement supérieur chinois, qui fait que l'internationalisation est peut être autant guidée par l'appât du gain que par les politiques officielles. Par ailleurs, une expérience de ce modèle montre que parfois, enseignants et administrateurs se disent «fatigués » de suivre des instructions officielles contradictoires au fil des ans, et font preuve d'une créativité assez éloquente pour résister. Les différentes stratégies mises en place par les universités peuvent par ailleurs mener à des phénomènes surprenants. L'un d'eux est le développement très rapide des départements universitaires de français (Bel, 2014). Vu de l'extérieur, ce développement peut être vu comme logique, résultant de la volonté du gouvernement de s'implanter en Afrique francophone et de s'en donner les moyens en formant des jeunes chinois à l'apprentissage du français. Si cela peut être un élément d'explication, il reste très secondaire et vient loin derrière d'autres, tels que la massification de l'enseignement supérieur chinois, la volonté des universités de se donner (à bon prix) une image de modernité à travers le développement de l'enseignement de langues au capital d'image très élevé (comme le français) ou encore la politique des provinces, qui jouent maintenant un rôle majeur pour autoriser administrativement l'ouverture de nouvelles spécialités. En revanche, les débouchés en Afrique sont une conséquence heureuse pour les étudiants comme pour les universités (beaucoup de spécialités auraient déjà fermé, faute de débouchés pour leurs étudiants).

On peut conclure de ces différents éléments que les choix des acteurs impliqués constituent un fondement incontournable de la dynamique d'ensemble. Concernant les étudiants par exemple, peu avaient prévu d'aller travailler en Afrique et si les candidats ne se bousculent pas, les opportunités d'emplois sont réelles comme le sont les

Recherches en didactique des langues et des cultures, 12-1 | 2015 
avantages (salaires, bénéfices divers....). De plus, l'élément distinctif et valorisable (donc monnayable) sur le marché du travail est essentiellement la connaissance de la langue française. La règle non écrite du marché du travail chinois, qui veut qu'on trouve beaucoup plus facilement un emploi si on vient d'une très bonne université et / ou si on a des relations, joue beaucoup moins dans ce cas là. Mais toutes ces migrations sont en grande majorité économiques : rester en Afrique quelques années de façon à accumuler un capital et profiter des avantages offerts au retour (par exemple un houkou ${ }^{27}$ permettant de s'établir dans une grande ville). Ces stratégies d'étudiants sont aussi des stratégies familiales.

Toute vision proposant un gommage des initiatives individuelles contribue à avoir une vision biaisée, notamment des stratégies à l'œuvre. La politique d'ouverture ainsi que le développement d'un capitalisme farouche ont entrainé une démultiplication de forces vives suivant leur propre agenda, tout en donnant l'impression d'être en ligne avec celui du gouvernement.

L'offre du français à l'UNCS comme du mandarin au Cameroun relèvent donc d'effets d'opportunités, que ce soit du point de vue institutionnel (la politique d'influence) que des universités (afficher une image valorisée) ou bien des différents individus qui s'y impliquent sous des formes diverses (développer des compétences dans des buts professionnels, à plus ou moins longs termes).

\section{Conclusion}

Cette réflexion est partie de l'idée d'une rencontre non prévue, celle de la francophonie dans le cadre de la Chinafrique. Elle nous a permis de remarquer les liens peu évidents entre la Chine et la francophonie sinon à travers l'enseignement du français langue étrangère. Des discours d'étudiants et de chercheurs nous ont également révélé les représentations qui fondent parfois les projets avec la Chine ou bien l'Afrique, des stéréotypes qui détonnent avec l'intensification croissante des activités économiques entre la Chine et l'Afrique. Cette dynamique a pourtant des conséquences sur le plan académique, puisqu'elle va imposer l'Afrique et la francophonie dans les projets académiques d'universités chinoises. Cela a conduit à des questionnements qui ont permis de mettre en évidence quelques-uns des enjeux de la Chinafrique. En tant que politique d'influence, certaines stratégies développées traduisent une politique d'expansion de la Chine en Afrique, présentée du point de vue chinois comme du «donnant-donnant ». En même temps, les processus rencontrés traduisent également des projections plus situées des acteurs impliqués dans ces relations, à l'affut de gains ou suivant leurs propres agendas sous des formes variées.

La Chinafrique projette donc la diversité et les langues avec, en arrière-plan, l'idée de l'expansion et de la diffusion de valeurs liées à la Chine. Les tentatives tièdes d'appel à la diversité équivalent en fait à du saupoudrage qui consiste à nier les expériences d'acteurs impliqués dans ces constructions. Que le projet d'ensemble soit politique ou non, il nous semble en tout cas qu'une prise en compte des expériences des acteurs pourrait permettre de valoriser des points de vue plus situés. Ce qui ne peut être possible que par un changement d'angle d'analyse, en adoptant par exemple celui de chacun des acteurs qui profitent des projets élaborés. 


\section{BIBLIOGRAPHIE}

Bel D. (2014). « L'enseignement du français en Chine » in Wolff A. (dir.), La langue française dans le monde, Paris, OIF / Nathan, 290-322.

Bel D. (2014). « Universités de classe mondiale : le cas chinois

Une vision systémique », In : Mario Laforest, Gilles Breton et David Bel (dir.), Réflexions sur l'internationalisation du monde universitaire: Points de vue d'acteurs, Cahier $\mathrm{n}^{\circ} 1$ du Réseau international sur la mondialisation de l'enseignement supérieur (RIMES), Paris, EAC, 29-53.

Cabestan J.-P., (2013). « Les relations Chine-Afrique: nouvelles responsabilités et nouveaux défis d'une puissance mondiale en devenir », Hérodote $3 f\left(n^{\circ} 150\right), 150-171$.

Chaubet F. (2004). « L'Alliance française ou la diplomatie de la langue (1883-1914)», Revue historique, vol. $4 \mathrm{n}^{\circ} 632,763-785$.

Chen J. (2008). « La formation des bénévoles pour les Jeux olympiques de Beijing, une inspiration pour la conception des programmes d'enseignement de français ", Synergies Chine 3, 59-66.

Deniau X. (2003). La Francophonie, Paris, QSJ.

Hayhoe R. et al. (2011). Portraits of 21st Century Chinese Universities : In the Move to Mass Higher Education, Hong Kong: Comparative Education Research Centre, University of Hong Kong and Springer.

Hu Y. (2006). «Qu'est-ce qui fait courir les étudiants chinois vers la France ? » in Synergies Chine 1, 192-199.

Li H. (2007). « L'expérience de la conception d'un cours sur la francophonie », Synergies Chine 2, 125-130.

Li H. (2010). « Enseigner la francophonie avec quel manuel ? », Synergies Chine 5, 71-80.

Pernet-Liu A. (2013). « Les sujets de mémoires d'étudiants chinois: choix personnel et culture universitaire », Synergies Chine 8, 177-184.

Ricard A., Sur la Francophonie, vrai (mais inavouable) héritage positif de la colonisation?, Repères DoRiF n. 2 Voix/voies excentriques: la langue française face à l'altérité - volet n. 1 - novembre 2012 - Les francophonies et francographies africaines face à la référence culturelle française.

Robillard, D. de, Beniamino, M. (1993). Le français dans l'espace francophone, Honoré Champion, Paris.

Valdman A. (1979). Le français hors de France, Paris, Champion.

Wolton D. (2012). « Conclusion » in Nowicki et Mayaux (dir.), L'autre francophonie, Honore Champion, Paris, 326-329.

Xie Y. (2008). Représentations de la France et des Français, trajectoires et construction d'une compétence interculturelle en contexte sino-français, Thèse de doctorat, Université Paris III-Sorbonne Nouvelle.

\section{NOTES}

1. Il faut, ceci dit, se garder ici d'une vision strictement européano-centrée. Effectivement, il est assez courant, en chinois, de juxtaposer le premier caractère de deux expressions (dans le cas 
présent: 中非) ce qui pourrait donner, en traduction littérale, Chine-Afrique, voire même Chinafrique, pour désigner les relations entre la Chine et les pays du continent africain.

2. La spécialité de français a été ouverte en 2007. D'abord dépendante administrativement du Campus de Nanhai (qui avait, à ce moment-là, à la fois le statut aussi hybride que curieux de campus et de faculté autonome), elle a été rattachée en septembre 2013 à l'une des quatre nouvelles facultés ouvertes cette année-là par l'Université Normale de Chine du Sud: l' International Business College.

3. «Didactique du français, formation et diversité/altérité en contextes universitaires ": pratiques, représentations et évolutions dans différents environnements " (pour les détails, voir l'introduction de ce numéro), ainsi que : http://projetauf.wix.com/diffodia\#

4. Il faut préciser qu'au sein de l'université existaient déjà des spécialités d'anglais, russe et japonais. C'est donc dans le contexte d'ouverture vers d'autres langues que le français a été choisi.

5. Ce choix de se focaliser sur l'Afrique plutôt qu'une autre francophonie (canadienne, asiatique, aire créole...) s'explique par la volonté, à travers un tel cours, de déconstruire les stéréotypes circulant sur l'Afrique chez des étudiants qui pourraient être amené à y travailler.

6. Elle évoque des "relations proches, des valeurs communes» (sic) entre les Français et les Chinois.

7. Dans le cadre de réalisation de dossiers, les étudiants devaient dresser le portrait et interroger des Francophones non originaires de France. En plus d'Africains, on aurait pu en évoquer qui viennent du Proche-Orient.

8. Dans le contexte chinois, cette décision est inhabituelle et même audacieuse.

9. Dans cette situation, la représentation de l'étranger (i.e. de l'Occidental) comme plus efficace en tant que directeur d'un département est partagée: on pense en effet qu'il consacrera la majeure partie du temps à travailler pour le développement dudit département et non à " faire du relationnel » (ce que feraient les Chinois). Cela renvoie à l'image idéalisée de la politique d'ouverture, lancée par Deng Xiaoping, qui affirme que le recours à l'étranger-expert est un moyen efficace pour le développement du pays.

10. Voir sur : http://www.frontenac-ameriques.org/la-francophonie-en-amerique/article/quest-ce-que-la-francophonie.

11. Ont été interrogés des apprenants de chinois à l'Institut Confucius (désormais IC) de Yaoundé au Cameroun.

12. Robillard et Beniamino (1993).

13. Synergies Chine est coordonnée par une équipe d'enseignants-chercheurs chinois avec l'aide de collègues français et est partiellement financée par l'Ambassade de France en Chine. Le corpus est constitué d'articles issus des huit premiers numéros de cette revue, depuis son lancement en 2006 jusqu'au n ${ }^{\circ} 8$ publié en 2013 , soit un total de près de 170 articles.

14. Sur les 8 numéros : 148 occurrences dans 25 articles différents. Il apparaît peu à chaque fois sauf dans les deux articles de Li H. (moitié des occurrences).

15. Ce qu'on retrouve par exemple en littérature pour prendre cet exemple: la littérature française et la littérature francophone sont deux domaines bien différenciés dans les universités françaises (et même dans les rayonnages des librairies et des bibliothèques !).

16. Curriculums et évaluation.

17. 外交学院法国与法语国家研究中心.

18. 复旦大学法语国家研究中心.

19. L'Université des langues étrangères et l'Université des langues et des cultures.

20. D'autres grands événements académiques viennent confirmer cette myopie, comme les Séminaire interculturels sino-français de Canton, organisés tous les deux ans.

21. Publié en 1988 pour le cycle de base ( $1^{\text {re }}$ et $2^{\text {e }}$ années), et en 1997 pour le cycle de perfectionnement ( $3^{\mathrm{e}}$ et $4^{\mathrm{e}}$ années). 
22. Nous avons parcouru les sites Internet de 60 universités ayant une spécialité de français (soit environ $40 \%$ du total), et notamment la page qui présente aux élèves du secondaire et candidats les spécialités offertes.

23. http://english.hanban.org/node_10971.htm (consulté le 2 janvier 2015).

24. Le Hanban est l'organisme ministériel chinois en charge des Instituts Confucius.

25. Le chinois est également enseigné à l'Université de Dschang et constitue un Département autonome à l'Université de Maroua qui forme par ailleurs de futurs enseignants destinés aux lycées du Cameroun où le chinois est désormais offert comme langue vivante à côté de l'espagnol et de l'allemand.

26. Il y a en Chine de très nombreuses langues, parlées soit par les minorités, soit par la majorité Han. On peut citer comme exemple le cantonais (langue du Guangdong, de Hong-Kong, de Macao, et d'une majeure partie de la diaspora chinoise).

27. Le droit de s'établir dans une ville en Chine.

\section{RÉSUMÉS}

Le terme «Chinafrique » est de nos jours largement utilisé pour caractériser les relations entre la Chine et le continent africain notamment dans le domaine économique. Avec cet article, les auteurs interrogent la Chinafrique dans sa dimension académique. S'appuyant sur leurs expériences de vie et de situations universitaires et sur un corpus d'entrevues réalisées en Chine et au Cameroun, ils mettent en évidence une rencontre non prévue, une rencontre fortuite avec la francophonie. Cette rencontre n'est néanmoins pas dénuée d'enjeux qui peuvent se décliner sous la forme de politique d'influence et d'effets d'opportunités notamment.

The term Chinafrica is nowadays widely used and refers to relationships between China and Africa, especially in the economic field. With this article, the two authors question the academic dimension of this relation. Based on their academic situations and life experiences and interviews conducted in China and Cameroon, they bring out an unexpected encounter with francophonie. However numerous issues are at stake, from political influence to opportunity effects regarding actors in various situations.

\section{INDEX}

Mots-clés : Chinafrique, université, acteurs, francophonie

Keywords : Chinafrica, academic relations, francophonie, actors

\section{AUTEURS}

\section{DAVID BEL}

En poste à l'Université Normale de Chine du Sud, à Canton, depuis 2005 (enseignant de FLE et responsable de département), David Bel est titulaire d'une maitrise en histoire de l'Université Paris-Ouest (Nanterre-La Défense) et d'un Master 2 en didactique du FLE / FOS de l'Université de 
Bourgogne. Il termine actuellement un doctorat à l'Université de Montréal. Dans sa thèse, il propose une analyse d'économie politique du développement de l'enseignement du français en Chine au niveau universitaire. Ses travaux de recherche portent également sur l'internationalisation de l'enseignement supérieur chinois et sur la francophonie. Courriel : davidbelnanhai[at]live.cn

Adresse : South China Normal University, Vice-Dean of International Business College. In charge of international relations and programs development.

\section{VALENTIN FEUSSI}

Université François-Rabelais de Tours, E.A. 4246 PREFics-Dynadiv

Maitre de conférences en Sociolinguistique et didactique des langues à l'Université François Rabelais de Tours, E.A. 4246 PREFics-Dynadiv, après avoir exercé des fonctions similaires à l'Université de Douala au Cameroun, Valentin Feussi mène actuellement des recherches sur les francophonies plurielles qu'il interroge d'un point de vue sociolinguistique, didactique et historique.

Courriel : valentin.feussi[at]univ-tours.fr Adresse : Université François-Rabelais, UFR Lettres et Langues, Département Sociolinguistique et Didactique des Langues, 3 rue des Tanneurs, 37000 Tours, France. 